\title{
Short-Time Flare Events of EV Lac
}

\author{
B.E. Zhilyaev, I.A. Verlyuk \\ Main Astronomical Observatory, National Academy of Sciences of Ukraine, \\ $252650 \mathrm{Kiev}$, Ukraine
}

\section{Observations}

Moffett (1972) pointed out the existence of short-time outbursts in flare stars as long ago as 1972. Gershberg \& Petrov (1986) had registered a flare in EV Lac with a duration of $2^{\mathrm{s}} .4$ and an amplitude of $3^{\mathrm{m}} \cdot 1$ in 1984 with $A S T R O N$. In recent years, a few reports appeared about observations of short-time outbursts in EV Lac with a duration in the range from 0.1 to several seconds. These results were summarized briefly by Zhilyaev (1993). Our observations confirm the existence of short-time flare activity on EV Lac.

The observations reported here were obtained at the $60 \mathrm{~cm}$ telescope at the Peak Terskol high-altitude Observatory ( $3100 \mathrm{~m}$ above sea level) with a highspeed two-channel photometer (Zhilyaev et al. 1992) in $B$ as well as simultaneously in both, $U$ and $V$ bands, with a time resolution down to 0.01 . A new prefiltering technique (Zhilyaev 1995) has been used for the detection of smallscale activity of the flare star EV Lac.

\section{Results}

1. The time span of the detected flare events lies in the subsecond range down to $0^{\mathrm{s}} .1$ (Zhilyaev et al. 1990). Fig. 1 represents an extreme short-term flare in $U$ and $V$ bands simultaneously, with a duration of about 0.3 and with amplitudes of $\Delta V \approx 0.8, \Delta U \approx 3^{\mathrm{m}}$.

2. The above-mentioned new prefiltering technique for high time resolution data allows to detect an event amplitude comparable with that caused by counting statistics. An example of such a small-scale flare with 1.5 duration and $0^{\mathrm{m}} 4$ amplitude can be seen in Fig. 1 of Zhilyaev (1995).

3. A new kind of flares was detected in EV Lac. A few events show a flare in the $U$ band accompanied by a dip in the $V$ band at the same time. Fig. 2 represents such an event with a duration of about $10^{\mathrm{B}}$ and amplitudes in $U$ and $V$ of $-1^{\mathrm{m}} \cdot 2$ and $+0^{\mathrm{m}} .05$, respectively. 


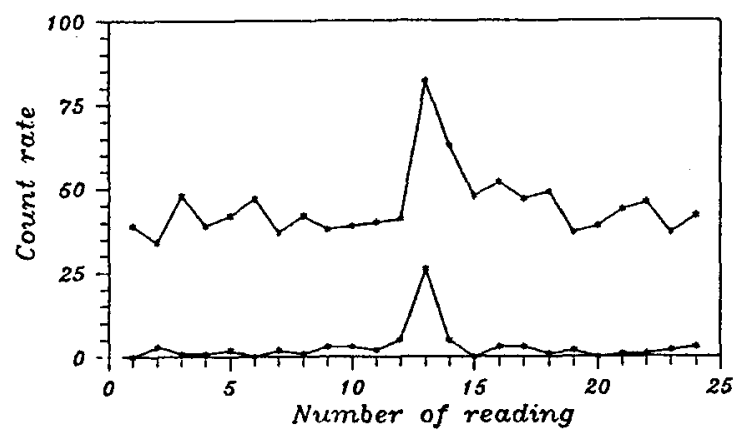

Fig. 1. EV Lac on 1990 Aug. 26, 00:04 UT. Time resolution: 0.05. $V$ band (top), $U$ band (bottom).
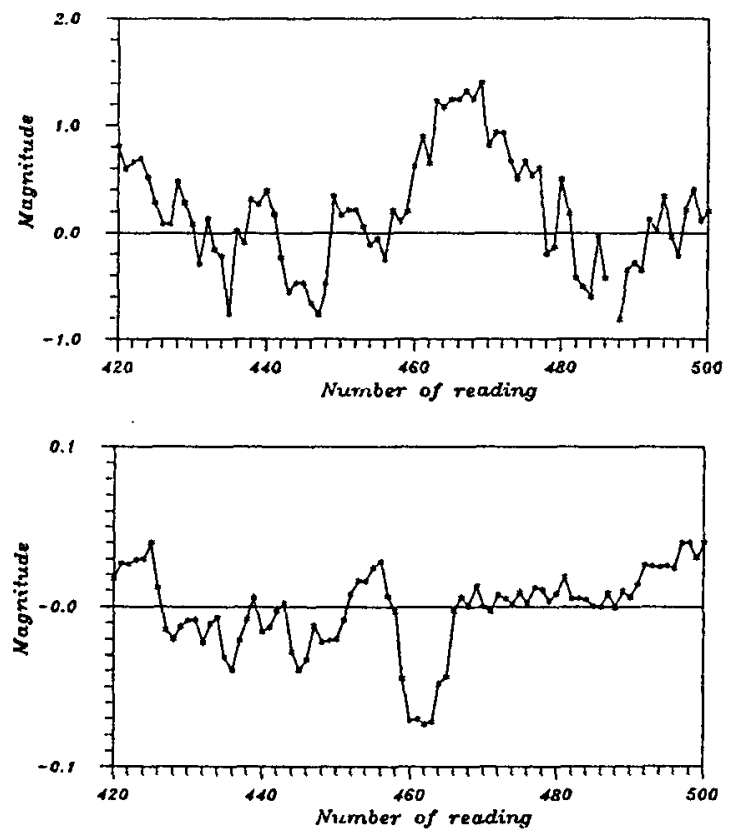

Fig. 2. EV Lac on 1990 Aug. 27, 00:45 UT. Time resolution: 0.05. U band (top), $V$ band (bottom). Prefiltering technique was used.

\section{References}

Gershberg R.E., Petrov P.P., 1986, Flare Stars and Related Objects, Publishing House of Acad. of Sci., Yerevan, p. 38

Moffett Y.J., 1972, Nature 240, 41

Zhilyaev B.E., Romaniuk Ya.O., Svyatogorov O.A., 1990, IAU Symp. 137, 35

Zhilyaev B.E., Romaniuk Ya.O., Svyatogorov O.A., 1992, AZh 69, 895

Zhilyaev B.E., 1993, Kinematics and Physics of Celestial Bodies, 10, 32

Zhilyaev B.E., 1995, this volume, p. 133 